\title{
COVID-19 Pandemic: Mental Health of Doctors in Bangladesh
}

\author{
Roufun Naher ${ }^{1}$, Mamun Al Mahtab ${ }^{2 *}$, Sheikh Mohammad Fazle \\ Akbar $^{3}$ and Nurul Islam Hasib ${ }^{4}$ \\ ${ }^{1}$ Department of Educational and Counselling Psychology, University of Dhaka, \\ Bangladesh \\ ${ }^{2}$ Department of Hepatology, Bangabandhu Sheikh Mujib Medical University, Dhaka, \\ Bangladesh \\ ${ }^{3}$ Department of Gastroenterology and Metabology, Ehime University Graduate \\ School of Medicine, Ehime, Japan \\ ${ }^{4}$ Bangladesh Post, Dhaka, Bangladesh \\ *Corresponding Author: Mamun Al Mahtab, Professor, Chairman, Department of \\ Hepatology, Bangabandhu Sheikh Mujib Medical University, Dhaka, Bangladesh.
}

Received: February 11, 2021

Published: March 20, 2021

(C) All rights are reserved by Mamun Al

Mahtab., et al.

\begin{abstract}
Background: The COVID-19 pandemic has affected everyone's life in all aspects. It presents an unprecedented challenge to public health. Doctors and other healthcare providers are battling the virus on the frontline.

Objective: This study is to see the mental health conditions of doctors in Bangladesh during the COVID-19 pandemic as well as to identify the percentage of doctors who are at high mental health risk and thus, need clinical intervention.

Method: We have assessed the mental health of 358 Dhaka-based doctors applying 'General Health Questionnaire-12' in an online survey.

Results: The study shows that a significant number of doctors (78.5\%) are at risk of mental health conditions. They score high in psychological distress, which means that their psychological wellbeing in the ongoing COVID-19 pandemic is lower than usual. Genderwise, it is also found that psychological wellbeing of female doctors is lower than their male counterparts.

Conclusion: The results of this study support the other similar studies conducted in different countries during the COVID-19 pandemic which indicate that doctors and health care professionals are at high risk of mental health. The results also highlight the importance of ensuring psychological support and mental health care to Bangladeshi doctors during crisis periods.
\end{abstract}

Keywords: COVID-19 Pandemic; Doctors; Mental Health; Bangladesh

\section{Introduction}

Uncertainty, lockdown and economic crisis resulting from COVID-19 pandemic could increase the risk of mental health issues and worsen the health inequalities [1]. COVID-19 places additional pressure on the entire healthcare system including doctors, who experience high levels of work stress even in normal circumstances, and research shows that such additional pressure puts doctors 
at greater risk of psychological distress [2]. Researchers found high prevalence of stress, anxiety and psychological distress especially among female doctors, young health care workers and those who provided services to COVID-19 patients or suspected COVID-19 patients [3]. In a survey, out of the 906 healthcare workers 48 (5.3\%) were screened positive for moderate to very-severe depression, $79(8.7 \%)$ for moderate to extremely-severe anxiety, 20 (2.2\%) for moderate to extremely-severe stress, and 34 (3.8\%) for moderate to severe levels of psychological distress [4]. It is also found that depressive and anxiety symptoms are more common among the healthcare professionals who are less psychologically prepared, lacking perceived self-efficacy, lacking family support, and those with poor sleep quality $[5,6]$. Similarly, researchers also warn of increasing rates of social problems such as domestic violence, suicide and substance abuse during the pandemic $[7,8]$. Despite the resilience of healthcare professionals, a large number of them has experienced and will experience some sort of physical and psychological difficulties $[9,10]$.

The impact of COVID-19 pandemic in Bangladesh is similar to other countries of the world. The government's disease monitoring agency, IEDCR, identified the first three cases on the $8^{\text {th }}$ March 2020. Like other countries, doctors in Bangladesh are the frontline fighters in COVID-19 crisis. Thus, their mental health, emotional safety and wellbeing matter. Moreover, in crisis period like COVID-19 pandemic, doctors are crucial part of overall health care system and their service is utterly dependent on their mental health and wellbeing. Different types of unprocessed and difficult emotions such as stress, anger, sadness, grief can make them more vulnerable. Researchers found that these emotions can compromise doctors' personal wellbeing, since suppressed or unprocessed emotions may lead to burnout, moral distress, compassion fatigue, and poor clinical decisions which adversely affect patient care [1113]. Therefore, at least a primary mental health assessment was needed to understand the mental health risk of Bangladeshi doctors during the COVID-19 pandemic for creating awareness, and taking measures such as ensuring mental health care, treatment and policy support.

\section{Aim of the Study}

This research aimed to investigate the psychological impact of COVID-19 pandemic on Dhaka based doctors and to identify the percentage of doctors who are at high risk of mental health which can be considered as clinical cases, and thus, require clinical attention.

\section{Materials and Methods}

The cross-sectional survey design was followed for this study. We started the survey in August 2020 and finished data collection in November 2020. The research was approved by the research ethics committee of Department of Educational and Counselling Psychology, University of Dhaka.

\section{Participants}

Initially 416 doctors of Dhaka city participated in this study; 358 doctors' responses were included and the rest 58 incomplete responses were excluded. Among the 358 participants, $63.1 \%$ were male and $36.9 \%$ were female. Doctors from all age groups participated in this study, for example, $24.3 \%$ doctors were from the age group 20 - 30, 50.3\% were from 31 - 40, 19\% were from 41 - 50 and $6.4 \%$ were from 51 - 65. In terms of marital status, $79.6 \%$ doctors were married, $18.4 \%$ were unmarried, and $2 \%$ were divorced or separated from their spouses. Doctors from different type of medical setting attended in this study such as $30.7 \%$ doctors were from covid dedicated hospital, $16.5 \%$ of them were from non-covid hospital, $47.2 \%$ were from mixed type hospital (both covid and noncovid) and 5.6\% doctors were from others type of workplace. The duration of the survey was 3 months (from August 2020 to November 2020) and in this timeline $28.8 \%$ doctors reported that they have already recovered from COVID-19.

\section{Measures}

Demographic information: We collected information about the doctors' gender, age, marital status, type of workplace during COVID-19 pandemic and the information whether they have been affected by COVID-19 or not.

General health questionnaire-12 (GHQ-12): As an investigation tool the Bangla translated version of the 12-item General Health Questionnaire (GHQ-12) was used. This questionnaire was originally developed as a 60-item questionnaire. GHQ-12 consists of 12 items which is related to mental health such as recent feelings, behavior, mood rather than physical health $[15,16]$. The items of the questionnaire phrased in both positive and negative directions. The answers were organized in four-point Likert scales. However, the response options are phrased in terms of 'less than usual', 'not 


\begin{tabular}{|l|c|}
\hline \multicolumn{1}{|c|}{ Characteristics } & n (\%) \\
\hline Gender & \\
Male & 63.1 \\
Female & 36.9 \\
\hline Age range & \\
20 - 30 & 24.3 \\
31 - 40 & 50.3 \\
41 - 50 & 19 \\
51 - 65 & 6.4 \\
\hline Marital status & \\
Married & 79.6 \\
Unmarried & 18.4 \\
Divorced/ widowed/separated & 2 \\
\hline Type of workplace & \\
Covid dedicated hospital & 30.7 \\
Non-covid hospital & 16.5 \\
Mixed type (covid, non-covid both) & 47.2 \\
Other type of workplace & 5.6 \\
\hline Corona virus infection & 28.8 \\
Already infected & 72.2 \\
Not infected & \\
\hline
\end{tabular}

Table 1: Demographic characteristics of the participants.

more than usual', 'rather more than usual', or 'much more than usual'. These are already arranged, so no reverse scoring is needed. GHQ-12 is a self-administered questionnaire focuses on two major areas - appearance of new and distressing phenomena and inability to carry out normal functions. For this study GHQ-12 was used as this is recognized as an ideal and widely used screening device for identifying non-psychotic and minor psychiatric disorders to help inform further intervention. The default scoring of GHQ-12 ranges from $0-3$, with a maximum score of 36 , and higher scores reflect higher distress or psychiatric illness. As an indication of 'caseness' or clinical attention the suggested default threshold for GHQ-12 is 11/12 (maximum score 36) in liker form.

\section{Procedure}

We utilized the data of an ongoing study for this research. It was an online survey and each participant was provided with a google form via email, whatsapp, viber, and messenger. The google form was consists of three parts such as the written consent paper along with the instruction for the participants, the demographic information of the participants and the mental health related questionnaire (GHQ12) respectively. The participants were instructed to complete and submit the google form online. Each participant required 10 - 15 minutes approximately to fill up the form. As GHQ-12 is an easily understandable self-report measure, the participants did not report any issues or concerns while filling up the google form. They were provided with the contact number and email address of one of the investigators and requested to communicate if needed. The participants were also requested to inform if they feel discomfort or become emotionally vulnerable during or after the data collection. Mental health first aid service was ready and available for them.

\section{Results}

The participants' obtaining GHQ-12 scores showed that 78.5\% doctors' psychological distress during COVID-19 pandemic is more or much more than usual and they scored above the suggested GHQ-12 threshold. In table 2 we can see that only $21 \%$ doctors experienced usual or less than usual psychological distress, whereas $58.9 \%$ doctors experienced more than usual and 19.6\% doctors experienced much more than usual psychological distress.

The results also indicate that psychological distress of female doctors is higher than the male doctors. Table 3 shows that the mean score of female doctors is 19.23 and male doctors is 17.67. Thus, female doctors scored higher in GHQ-12 than the male doctors and the difference was considered statistically significant $(\mathrm{t}$ $=2.011, \mathrm{p}=.045$ ). It is also shown that the mean GHQ-12 score of both male and female doctors are above the suggested threshold which indicate possible psychological distress or disturbance.

\section{Discussion}

The objective of this study was to see the prevalence of psychological distress among Bangladeshi doctors during the Covid-19 pandemic. Our findings indicate that the psychological wellbeing of majority of doctors (78.5\%) was poor as they crossed the threshold level of the GHQ-12 score. Therefore, a large number of doctors experienced concerning level of psychological distress or disturbances during Covid-19 pandemic which can be considered as clinical cases, and thus, need mental health care or clinical intervention. 


\begin{tabular}{|l|c|c|c|}
\hline $\begin{array}{c}\text { GHQ-12 } \\
\text { score }\end{array}$ & $\begin{array}{c}\text { Number of } \\
\text { doctors }\end{array}$ & $\begin{array}{c}\text { \% of the } \\
\text { doctors }\end{array}$ & $\begin{array}{c}\text { \% of above } \\
\text { threshold GHQ-12 } \\
\text { score of the } \\
\text { participants }\end{array}$ \\
\hline $0-11$ & 77 & 21.5 & \\
\hline $12-24$ & 211 & 58.9 & 58.9 \\
\hline $25-36$ & 70 & 19.6 & 19.6 \\
\hline & $\mathrm{n}=358$ & & Total $=78.5 \%$ \\
\hline
\end{tabular}

Table 2: Prevalence of psychological distress.

*The suggested threshold score of GHQ-12 is 11/12. Equal or above this score indicate possible psychological distress and needs clinical intervention.

${ }^{*}$ GHQ-12 score 0-11 indicates usual or less than usual psychological distress.

*GHQ-12 score 11-24 indicates more than usual psychological distress.

${ }^{*}$ GHQ-12 score 25-36 indicates much more than usual psychological distress.

\begin{tabular}{|l|c|c|c|c|c|}
\hline \multicolumn{1}{|c|}{ Variable } & & $\begin{array}{c}\text { Females } \\
(\mathbf{n = 4 4 )}\end{array}$ & $\begin{array}{c}\text { Males } \\
(\mathbf{n = 3 1 )}\end{array}$ & $\begin{array}{c}\text { t- } \\
\text { value }\end{array}$ & prob \\
\hline $\begin{array}{l}\text { GHQ Score } \\
\text { (psychological } \\
\text { distress) }\end{array}$ & M & $\begin{array}{c}19.23 \\
\text { SD }\end{array}$ & $\begin{array}{c}17.67 \\
(7.200)\end{array}$ & 2.011 & .045 \\
\hline
\end{tabular}

Table 3: Comparison of psychological wellbeing by gender.

The findings also showed that female doctors are more vulnerable than their male counterparts in terms of mental health and wellbeing, though both groups scored high in psychological distress. We utilized the data of an ongoing study named social support and psychological wellbeing of Bangladeshi doctors during COVID-19 pandemic, thus, we are on the way of exploring and addressing the factors contributing this concerning level of psychological distress among Bangladeshi doctors. A plenty of research evidence showed that excessive work pressure, inadequate self-care, lack of professional help seeking behavior, sense of insecurity and inadequate PPE, lack of social support, physical distancing from family members during pandemic make doctors and health professional more vulnerable [17-20].
However, from risk management perspective, it's crucial to manage work stress, ensure social as well as professional support from individual and organizational level. Similarly, it is evident that professionals' motivation and morale are significantly improved when they perceive that their efforts are recognized and reciprocated by employers and authorities [21,22].

Further studies are needed to explore the factors of high mental health risk of Bangladeshi doctors during the pandemic and to devise policy from risk management perspective. Convenient sampling technique was used and self-report assessment tool was applied to collect data for this study. Thus, randomization and clinical interview are recommended for further studies.

\section{Conclusion}

The study suggests that doctors are at high risk of mental health during the Covid-19 pandemic.

This is consistent to other similar studies conducted in different countries during the ongoing pandemic. The results highlight the importance of ensuring psychological support and mental health care to Bangladeshi doctors during the crisis periods. Otherwise, healthcare services can be disrupted.

\section{Acknowledgements}

Gratitude to the participants who took time during this difficult period to fill up the form.

\section{Conflict of Interest}

There is no conflict of interest.

\section{Bibliography}

1. Moreno Carmen., et al. "How mental health care should change as a consequence of the COVID-19 pandemic". The Lancet Psychiatry 7.9 (2020): 813-824.

2. Galbraith Niall., et al. "The mental health of doctors during the COVID-19 pandemic". BJP Sych Bulletin (2020): 1-4.

3. Badahdah Abdallah., et al. "The mental health of health care workers in Oman during the COVID-19 pandemic". The International Journal of Social Psychiatry (2020): 20764020939596.

4. Chew Nicholas WS., et al. "A multinational, multicentre study on the psychological outcomes and associated physical symp- 
toms amongst healthcare workers during COVID-19 outbreak". Brain, Behavior, and Immunity 88 (2020): 559-565.

5. Du Jiang., et al. "Psychological symptoms among frontline healthcare workers during COVID-19 outbreak in Wuhan". General Hospital Psychiatry 67 (2020): 144-145.

6. Huang, Yeen and Ning Zhao. "Generalized anxiety disorder, depressive symptoms and sleep quality during COVID-19 outbreak in China: a web-based cross-sectional survey". Psychiatry Research 288 (2020): 112954.

7. Clay James M and Matthew O Parker. "Alcohol use and misuse during the COVID-19 pandemic: a potential public health crisis?" The Lancet Public Health 5.5 (2020): e259.

8. Reger Mark A., et al. "Suicide Mortality and Coronavirus Disease 2019-A Perfect Storm?" The Journal of the American Medical Association Psychiatry (2020).

9. Greenberg Neil., et al. "Managing mental health challenges faced by healthcare workers during covid-19 pandemic". BMJ 368 (2020): m1211.

10. Matheson C., et al. "Resilience of primary healthcare professionals working in challenging environments: A focus group study". British Journal of General Practice 66.648 (2016): e507e515.

11. Sanchez-Reilly Sandra., et al. "Caring for oneself to care for others: physicians and their self-care". The Journal of Supportive Oncology 11.2 (2013): 75-81.

12. Kearney Michael K., et al. "Self-care of physicians caring for patients at the end of life: "Being connected... a key to my survival". The Journal of the American Medical Association 301.11 (2009): 1155-1164.

13. Grunfeld E., et al. "Cancer care workers in Ontario: prevalence of burnout, job stress and job satisfaction". CMAJ: Canadian Medical Association Journal = Journal de l'Association Medicale Canadienne 163.2 (2000): 166-169.

14. Golderberg D and Williams P. "A user's guide to the General Health Questionnaire". Windsor, UK (1988): NFER-Nelson (1988).
15. The general health questionnaire.

16. Tam Cindy W C., et al. "Severe acute respiratory syndrome (SARS) in Hong Kong in 2003: stress and psychological impact among frontline healthcare workers". Psychological Medicine 34.7 (2004): 1197-1204.

17. Hassan Tariq M., et al. "A postal survey of doctors' attitudes to becoming mentally ill". Clinical Medicine 9.4 (2009): 327-332.

18. White Alfred., et al. "Barriers to Mental Healthcare for Psychiatrists". Psychiatric Bulletin 30.10 (2006): 382-384.

19. Devnani Mahesh. "Factors associated with the willingness of health care personnel to work during an influenza public health emergency: an integrative review". Prehospital and Disaster Medicine 27.6 (2012): 551-566.

20. Ma Hui., et al. "Role stress, social support and occupational burnout among physicians in China: a path analysis approach". International Health 12.3 (2020): 157-163.

21. Wong, Eliza LY., et al. "Healthcare workers' duty concerns of working in the isolation ward during the novel H1N1 pandemic". Journal of Clinical Nursing 21.9-10 (2012): 1466-1475.

22. Khalid Imran., et al. "Healthcare Workers Emotions, Perceived Stressors and Coping Strategies During a MERS-CoV Outbreak". Clinical Medicine and Research 14.1 (2016): 7-14.

\section{Assets from publication with us}

- Prompt Acknowledgement after receiving the article

- Thorough Double blinded peer review

- Rapid Publication

- Issue of Publication Certificate

- High visibility of your Published work

Website: www.actascientific.com/

Submit Article: www.actascientific.com/submission.php Email us: editor@actascientific.com

Contact us: +919182824667 